\title{
SQUARE-SUMMABLE STABILITY IN PARABOLIC VOLTERRA DIFFERENCE EQUATIONS
}

\section{B. Shi, Z. C. Wang, and J. S. Yu}

AbStract. We consider some linear and nonlinear parabolic Volterra difference equations of the forms

$$
\Delta_{2}\left(u_{m, n}-\sum_{j=1}^{\infty} q_{j} u_{m, n-r_{j}}\right)+\sum_{i=1}^{\infty} p_{i} u_{m, n-k_{i}}=R \Delta_{1}^{2} u_{m-1, n+1}
$$

and

$$
\Delta_{2}\left[h\left(u_{m, n}\right)-\sum_{j=1}^{\infty} q_{j} g\left(u_{m, n-r_{j}}\right)\right]+\sum_{i=1}^{\infty} p_{i} f\left(u_{m, n-k_{i}}\right)=R \Delta_{1}^{2} F\left(u_{m-1, n+1}\right)
$$

for $m=0,1, \ldots, M-1$ and $n=0,1, \ldots$, and we obtain several sufficient conditions for the square-summable stability and $\phi$-square-summable stability of the zero solution.

\section{Introduction}

Consider the linear parabolic Volterra difference equations of neutral type

$$
\begin{gathered}
\Delta_{2}\left(u_{m, n}-\sum_{j=1}^{\infty} q_{j} u_{m, n-r_{j}}\right)+\sum_{i=1}^{\infty} p_{i} u_{m, n-k_{i}}=R \Delta_{1}^{2} u_{m-1, n+1} \\
\quad \text { for } m=0,1, \ldots, M-1 \text { and } n=0,1, \ldots,
\end{gathered}
$$

with homogeneous von Neumann boundary conditions (NBC):

$$
\Delta_{1} u_{0, n}=\Delta_{1} u_{M, n}=0 \text { for } n=0,1, \ldots,
$$

and initial conditions (IC):

$$
u_{m, i}=\mu_{m, i} \quad \text { for } m=0,1, \ldots, M-1 \text { and } i=\ldots,-2,-1,0,
$$

and nonlinear parabolic Volterra difference equations of neutral type

$$
\begin{gathered}
\Delta_{2}\left[h\left(u_{m, n}\right)-\sum_{j=1}^{\infty} q_{j} g\left(u_{m, n-r_{j}}\right)\right]+\sum_{i=1}^{\infty} p_{i} f\left(u_{m, n-k_{i}}\right)=R \Delta_{1}^{2} F\left(u_{m-1, n+1}\right) \\
\text { for } m=0,1, \ldots, M-1 \text { and } n=0,1, \ldots,
\end{gathered}
$$

with $\mathrm{IC}(3)$ and NBC:

$$
\Delta_{1} F\left(u_{0, n}\right)=\Delta_{1} F\left(u_{M, n}\right)=0 \text { for } n=0,1, \ldots,
$$

where $\Delta_{1}, \Delta_{1}^{2}$, and $\Delta_{2}$ are forward partial difference operators (see, for instance, Kelley and Peterson [11]) such that $\Delta_{1} u_{m, n}:=u_{m+1, n}-u_{m, n}, \Delta_{1}^{2} u_{m, n}:=\Delta_{1}\left(\Delta_{1} u_{m, n}\right)$ and $\Delta_{2} u_{m, n}:=u_{m, n+1}-u_{m, n}$ for $m=0,1, \ldots, M-1, n=0, \pm 1, \pm 2, \ldots ; p_{i}, q_{j} \in \mathbf{R}=$

Received July 6, 1995, revised March 14, 1996.

1991 Mathematics Subject Classification: 39A10.

Key words and phrases: parabolic Volterra difference equations, asymptotic stability, squaresummable stability, $\phi$-square-summable stability, homogeneous von Neumann boundary conditions. 
$(-\infty, \infty), k_{i}, r_{j} \in\{0,1, \ldots\}$ for $i, j=1,2, \ldots ; \mu_{m, i} \in \mathbf{R}$ for $m=0,1, \ldots, M-1$ and $i=\ldots,-1,0 ; R \in[0, \infty)$ and $f, g, h, F \in C(\mathbf{R}, \mathbf{R})$ such that $f(0)=g(0)=h(0)=$ $F(0)=0$. Thus, $u_{m, n}=0$ for $m=1,2, \ldots, M-1$ and $n=0, \pm 1, \pm 2, \ldots$, is a solution of (4), which we call the zero solution. Throughout this paper, let $P:=\sum_{i=1}^{\infty} p_{i}>0$, $P^{*}:=\sum_{i=1}^{\infty}\left|p_{i}\right|, P^{\prime}:=\sum_{i=1}^{\infty} k_{i}\left|p_{i}\right|, P^{\prime \prime}:=\sum_{i=1}^{\infty} k_{i}^{2}\left|p_{i}\right|, Q^{*}:=\sum_{j=1}^{\infty}\left|q_{j}\right|$ and $Q^{\prime}:=$ $\sum_{j=1}^{\infty} r_{j}\left|q_{j}\right|$, and suppose that $P, P^{*}, P^{\prime}, P^{\prime \prime}, Q^{*}, Q^{\prime}<\infty$ and that

$$
\|\mu\|:=\sup \left\{\left|\mu_{m, i}\right| \quad \mid \quad m=0,1, \ldots, M-1 \quad \text { and } i=\ldots,-1,0\right\}<\infty .
$$

For the sake of convenience in proving the (unique) existence of solutions of (1) with the initial-boundary conditions (2) and (3), we let $u_{m, i}=0$ for $m<0, m>M+1$, and $i=0, \pm 1, \pm 2, \ldots$.

By a solution of (1)-(3) or (4), (5) and (3), we mean a sequence $\left\{u_{m, n}\right\}$ which is defined for $m=0,1, \ldots, M+1$ and $n=0, \pm 1, \pm 2, \ldots$ and which satisfies (1) or (4) for $m=1,2, \ldots, M-1$ and $n=0,1, \ldots$, satisfies $\mathrm{NBC}(2)$ or $(5)$ for $n=0,1, \ldots$, and satisfies $\operatorname{IC}(3)$ for $m=0,1, \ldots, M-1$ and $i=\ldots,-1,0$.

By using the method similar to that in Zhang, Liu, and Cheng [12] or simply by iterative calculation, it is easy to show that (1) or (4) has a unique solution for the given boundary and initial conditions satisfying (6) (see Appendix).

In the sequel, we only consider the solutions of (1) and (4) with the initial conditions satisfying (6).

Recently, the oscillation (see [4-6, 17, 21], also Yu and Cui's survey paper [20]) of delay partial differential equations has been widely studied, while Xie [16] considered the stability of partial differential equations. The oscillation (see $[1,13]$ ) and the stability (see $[9,10,15]$, see also Burton's books [2, 3]) for Volterra integrodifferential equations also have been extensively approached, while Gopalsamy and Weng [8] considered the stability of a neutral integrodifferential equation. It is well-known that the behavior of a differential equation and its discrete analogue can be quite different. For example, every solution of the logistic equation

$$
x^{\prime}(t)=r x(t)\left[1-\frac{x(t)}{K}\right]
$$

is monotonic. But its discrete analogue

$$
x_{n+1}=m x_{n}\left(1-x_{n}\right)
$$

has a chaotic solution when $m=4$ (see [11]). In addition, there is a difference between the oscillation of delay differential equations and discrete analogues; for example, see [18]. In the last few years, many mathematicians have been studying difference systems. But only a few studies (see [7, 14, 22]) are devoted to partial difference equations and Volterra difference equations; we [14] considered the stability for neutral Volterra difference equations.

Our aim in this paper is to obtain sufficient conditions, which are "sharp" in some sense, for the square-summable stability and $\phi$-square-summable stability in parabolic Volterra difference equations of neutral type. Our results generalize the corresponding results in $[14,19]$.

We now give some definitions which will be needed in this paper.

Definition 1.1. The zero solution of (1) or (4) is said to be asymptotically stable (AS) if every solution $\left\{u_{m, n}\right\}$ of (1) or (4) with IC satisfying (6) has the property

$$
\lim _{n \rightarrow \infty} u_{m, n}=0 \quad \text { for } \quad m=0,1, \ldots, M+1 \text {. }
$$


Definition 1.2. The zero solution of (1) or (4) is said to be square-summably stable (SSS) if every solution $\left\{u_{m, n}\right\}$ of (1) or (4) with IC satisfying (6) has the property

$$
\sum_{n=0}^{\infty} u_{m, n}^{2}<\infty \quad \text { for } \quad m=0,1, \ldots, M+1 \text {. }
$$

It is easy to see that SSS implies AS.

Definition 1.3. The zero solution of (1) or (4) is said to be $\phi$-square-summably stable ( $\phi$-SSS) if every solution $\left\{u_{m, n}\right\}$ of (1) or (4) with IC satisfying (6) has the property

$$
\sum_{n=0}^{\infty} \phi^{2}\left(u_{m, n}\right)<\infty \quad \text { for } \quad m=0,1, \ldots, M+1
$$

where $\phi \in C(\mathbf{R}, \mathbf{R})$ and $\phi \not \equiv 0$.

Note that SSS implies $\phi$-SSS, and $\phi$-SSS implies SCS if $|\phi(x)| \geq|x|$ for $x \in \mathbf{R}$. It also is obvious that $\phi$-SSS implies AS if $\phi(x)=0$ implies $x=0$.

\section{Equation (1)}

For (1), we have the following

Theorem 2.1. Assume that

$$
Q^{*}+\frac{1}{2} P+P^{\prime}<1
$$

Then the zero solution of (1) is SSS.

Proof. It is easy to show that

$$
\sum_{i=1}^{\infty} p_{i} u_{m, n-k_{i}}=P u_{m, n+1}-\Delta\left(\sum_{i=1}^{\infty} p_{i} \sum_{s=n-k_{i}}^{n} u_{m, s}\right) .
$$

Hence, we can rewrite (1) as

$$
\Delta_{2}\left(u_{m, n}-\sum_{j=1}^{\infty} q_{j} u_{m, n-r_{j}}-\sum_{i=1}^{\infty} p_{i} \sum_{s=n-k_{i}}^{n} u_{m, s}\right)=-P u_{m, n+1}+R \Delta_{1}^{2} u_{m-1, n+1} .
$$

Define a Liapunov sequence by

$$
V_{n}^{(1)}=\sum_{m=0}^{M+1}\left(u_{m, n}-\sum_{j=1}^{\infty} q_{j} u_{m, n-r_{j}}-\sum_{i=1}^{\infty} p_{i} \sum_{s=n-k_{i}}^{n} u_{m, s}\right)^{2}
$$

Then we have

$$
\begin{aligned}
\Delta V_{n}^{(1)}= & \sum_{m=0}^{M+1}\left(-P u_{m, n+1}+R \Delta_{1}^{2} u_{m-1, n+1}\right)\left(u_{m, n+1}+u_{m, n}\right. \\
& -\sum_{j=1}^{\infty} q_{j} u_{m, n+1-r_{j}}-\sum_{j=1}^{\infty} q_{j} u_{m, n-r_{j}}-P u_{m, n+1} \\
& \left.-2 \sum_{i=1}^{\infty} p_{i} \sum_{s=n+1-k_{i}}^{n} u_{m, s}-\sum_{i=1}^{\infty} p_{i} u_{m, n-k_{i}}\right) .
\end{aligned}
$$

(We define $\sum_{i=m}^{n} *=0$ if $m>n$ ). 
First, let us consider

$$
\begin{aligned}
& -P \sum_{m=0}^{M+1} u_{m, n+1}\left(u_{m, n+1}+u_{m, n}-\sum_{j=1}^{\infty} q_{j} u_{m, n+1-r_{j}}-\sum_{j=1}^{\infty} q_{j} u_{m, n-r_{j}}\right. \\
& \left.-P u_{m, n+1}-2 \sum_{i=1}^{\infty} p_{i} \sum_{s=n+1-k_{i}}^{n} u_{m, s}-\sum_{i=1}^{\infty} p_{i} u_{m, n-k_{i}}\right) \\
& =-P \sum_{m=0}^{M+1} u_{m, n+1}\left(u_{m, n+1}+u_{m, n}-\sum_{j=1}^{\infty} q_{j} u_{m, n+1-r_{j}}-\sum_{j=1}^{\infty} q_{j} u_{m, n-r_{j}}\right. \\
& -P u_{m, n+1}-2 \sum_{i=1}^{\infty} p_{i} \sum_{s=n+1-k_{i}}^{n} u_{m, s}+u_{m, n+1}-u_{m, n} \\
& \left.-\sum_{j=1}^{\infty} q_{j} u_{m, n+1-r_{j}}+\sum_{j=1}^{\infty} q_{j} u_{m, n-r_{j}}-R \Delta_{1}^{2} u_{m-1, n+1}\right) \\
& =\sum_{m=0}^{M+1}\left(-2 P u_{m, n+1}^{2}+2 P \sum_{j=1}^{\infty} q_{j} u_{m, n+1} u_{m, n+1-r_{j}}+P^{2} u_{m, n+1}^{2}\right. \\
& \left.+2 P \sum_{i=1}^{\infty} \sum_{s=n+1-k_{i}}^{n} u_{m, n+1} u_{m, s}+P R u_{m, n+1} \Delta_{1}^{2} u_{m-1, n+1}\right) \\
& \leq \sum_{m=0}^{M+1}\left[-2 P u_{m, n+1}^{2}+P \sum_{j=1}^{\infty}\left|q_{j}\right|\left(u_{m, n+1}^{2}+u_{m, n+1-r_{j}}^{2}\right)+P^{2} u_{m, n+1}^{2}\right. \\
& \left.+P \sum_{i=1}^{\infty}\left|p_{i}\right| \sum_{s=n+1-k_{i}}^{n}\left(u_{m, n+1}^{2}+u_{m, s}^{2}\right)+P R u_{m, n+1} \Delta_{1}^{2} u_{m-1, n+1}\right] \\
& =\sum_{m=0}^{M+1}\left\{-2 P\left[1-\frac{1}{2}\left(Q^{*}+P+P^{\prime}\right)\right] u_{m, n+1}^{2}+P \sum_{j=1}^{\infty}\left|q_{j}\right| u_{m, n+1-r_{j}}^{2}\right. \\
& \left.+P \sum_{i=1}^{\infty}\left|p_{i}\right| \sum_{s=n+1-k_{i}}^{n} u_{m, s}^{2}+P R u_{m, n+1} \Delta_{1}^{2} u_{m-1, n+1}\right\} \text {. }
\end{aligned}
$$

Let us now consider

$$
\begin{aligned}
& R \sum_{m=0}^{M+1}\left(u_{m, n+1}+u_{m, n}-\sum_{j=1}^{\infty} q_{j} u_{m, n+1-r_{j}}-\sum_{j=1}^{\infty} q_{j} u_{m, n-r_{j}}-P u_{m, n+1}\right. \\
& \left.\quad-2 \sum_{i=1}^{\infty} p_{i} \sum_{s=n+1-k_{i}}^{n} u_{m, s}-\sum_{i=1}^{\infty} p_{i} u_{m, n-k_{i}}\right) \Delta_{1}^{2} u_{m-1, n+1} \\
& =R \sum_{m=0}^{M+1}\left(u_{m, n+1}+u_{m, n}-\sum_{j=1}^{\infty} q_{j} u_{m, n+1-r_{j}}-\sum_{j=1}^{\infty} q_{j} u_{m, n-r_{j}}-P u_{m, n+1}\right. \\
& \quad-2 \sum_{i=1}^{\infty} p_{i} \sum_{s=n+1-k_{i}}^{n} u_{m, s}+u_{m, n+1}-u_{m, n}-\sum_{j=1}^{\infty} q_{j} u_{m, n+1-r_{j}} \\
& \left.\quad+\sum_{j=1}^{\infty} q_{j} u_{m, n-r_{j}}-R \Delta_{1}^{2} u_{m-1, n+1}\right) \Delta_{1}^{2} u_{m-1, n+1}
\end{aligned}
$$




$$
\begin{aligned}
& \leq 2 R \sum_{m=0}^{M+1} u_{m, n+1} \Delta_{1}^{2} u_{m-1, n+1}-2 R \sum_{j=1}^{\infty} q_{j} \sum_{m=0}^{M+1} u_{m, n+1-r_{j}} \Delta_{1}^{2} u_{m-1, n+1} \\
& \quad-P R \sum_{m=0}^{M+1} u_{m, n+1} \Delta_{1}^{2} u_{m-1, n+1}-2 R \sum_{i=1}^{\infty} p_{i} \sum_{s=n+1-k_{i}}^{n} \sum_{m=0}^{M+1} u_{m, s} \Delta_{1}^{2} u_{m-1, n+1} .
\end{aligned}
$$

Therefore, we get

$$
\begin{aligned}
\Delta V_{n}^{(1)} \leq & -2 P\left[1-\frac{1}{2}\left(Q^{*}+P+P^{\prime}\right)\right] \sum_{m=0}^{M+1} u_{m, n+1}^{2}+P \sum_{m=0}^{M+1} \sum_{j=1}^{\infty}\left|q_{j}\right| u_{m, n+1-r_{j}}^{2} \\
& +P \sum_{m=0}^{M+1} \sum_{i=1}^{\infty}\left|p_{i}\right| \sum_{s=n+1-k_{i}}^{n} u_{m, s}^{2}+2 R \sum_{m=0}^{M+1} u_{m, n+1} \Delta_{1}^{2} u_{m-1, n+1} \\
& -R \sum_{j=1}^{\infty} q_{j} \sum_{m=0}^{M+1} u_{m, n+1-r_{j}} \Delta_{1}^{2} u_{m-1, n+1} \\
& -2 R \sum_{i=1}^{\infty} p_{i} \sum_{s=n+1}^{n} \sum_{m=0}^{M+1} u_{m, s} \Delta_{1}^{2} u_{m-1, n+1} .
\end{aligned}
$$

By using a summation-by-parts formula and $\operatorname{NBC}(2)$ (here, we define $\Delta_{1} u_{i, n}=0$ for $i \leq 0$ and $i \geq M+1$ ), we get

$$
\begin{aligned}
& 2 R \sum_{m=0}^{M+1} u_{m, n+1} \Delta_{1}^{2} u_{m-1, n+1}=-2 R \sum_{m=0}^{M+1}\left(\Delta_{1} u_{m, n+1}\right)^{2} \\
& -2 R \sum_{j=1}^{\infty} q_{j} \sum_{m=0}^{M+1} u_{m, n+1-r_{j}} \Delta_{1}^{2} u_{m-1, n+1} \\
& =2 R \sum_{j=1}^{\infty} q_{j} \sum_{m=0}^{M+1} \Delta_{1} u_{m, n+1-r_{j}} \Delta_{1} u_{m, n+1} \\
& \leq R \sum_{m=0}^{M+1} \sum_{j=1}^{\infty}\left|q_{j}\right|\left[\left(\Delta_{1} u_{m, n+1}\right)^{2}+\left(\Delta_{1} u_{m, n+1-r_{j}}\right)^{2}\right] \\
& =R Q^{*} \sum_{m=0}^{M+1}\left(\Delta_{1} u_{m, n+1}\right)^{2}+R \sum_{m=0}^{M+1} \sum_{j=1}^{\infty}\left|q_{j}\right|\left(\Delta_{1} u_{m, n+1-r_{j}}\right)^{2} \\
& -2 R \sum_{i=1}^{\infty} p_{i} \sum_{s=n+1-k_{i}}^{n} \sum_{m=0}^{M+1} u_{m, s} \Delta_{1}^{2} u_{m-1, n+1} \\
& =2 R \sum_{i=1}^{\infty} p_{i} \sum_{s=n+1-k_{i}}^{n} \sum_{m=0}^{M+1} \Delta_{1} u_{m, s} \Delta_{1} u_{m, n+1} \\
& \leq R \sum_{m=0}^{M+1} \sum_{i=1}^{\infty}\left|p_{i}\right| \sum_{s=n+1-k_{i}}^{n}\left[\left(\Delta_{1} u_{m, s}\right)^{2}+\left(\Delta_{1} u_{m, n+1}\right)^{2}\right] \\
& =R P^{\prime} \sum_{m=0}^{M+1}\left(\Delta_{1} u_{m, n+1}\right)^{2}+R \sum_{m=0}^{M+1} \sum_{i=1}^{\infty}\left|p_{i}\right| \sum_{s=n+1-k_{i}}^{n}\left(\Delta_{1} u_{m, s}\right)^{2} \text {. }
\end{aligned}
$$


Hence, we get

$$
\begin{aligned}
\Delta V_{n}^{(1)} \leq- & 2 P\left[1-\frac{1}{2}\left(Q^{*}+P+P^{\prime}\right)\right] \sum_{m=0}^{M+1} u_{m, n+1}^{2} \\
& -2 R\left[1-\frac{1}{2}\left(Q^{*}+P^{\prime}\right)\right] \sum_{m=0}^{M+1}\left(\Delta_{1} u_{m, n+1}\right)^{2} \\
& +P \sum_{m=0}^{M+1} \sum_{j=1}^{\infty}\left|q_{j}\right| u_{m, n+1-r_{j}}^{2}+P \sum_{m=0}^{M+1} \sum_{i=1}^{\infty}\left|p_{i}\right| \sum_{s=n+1-k_{i}}^{n} u_{m, s}^{2} \\
& +R \sum_{m=0}^{M+1} \sum_{j=1}^{\infty}\left|q_{j}\right|\left(\Delta_{1} u_{m, n+1-r_{j}}\right)^{2}+R \sum_{m=0}^{M+1} \sum_{i=1}^{\infty}\left|p_{i}\right| \sum_{s=n+1-k_{i}}^{n}\left(\Delta_{1} u_{m, s}\right)^{2} .
\end{aligned}
$$

Now, define another Liapunov sequence by

$$
\begin{aligned}
V_{n}^{(2)}= & \sum_{m=0}^{M+1}\left[P \sum_{j=1}^{\infty}\left|q_{j}\right| \sum_{s=n+1-r_{j}}^{n} u_{m, s}^{2}+P \sum_{i=1}^{\infty}\left|p_{i}\right| \sum_{s=n+1-k_{i}}^{n} \sum_{t=s}^{n} u_{m, t}^{2}\right. \\
& \left.+R \sum_{j=1}^{\infty}\left|q_{j}\right| \sum_{s=n+1-r_{j}}^{n}\left(\Delta_{1} u_{m, s}\right)^{2}+R \sum_{i=1}^{\infty}\left|p_{i}\right| \sum_{s=n+1-k_{i}}^{n} \sum_{t=s}^{n}\left(\Delta_{1} u_{m, t}\right)^{2}\right] .
\end{aligned}
$$

Then, we obtain

$$
\begin{aligned}
\Delta V_{2}^{(2)}= & \sum_{m=0}^{M+1}\left\{P \sum_{j=1}^{\infty}\left|q_{j}\right|\left(u_{m, n+1}^{2}-u_{m, n+1-r_{j}}^{2}\right)+P \sum_{i=1}^{\infty}\left|p_{i}\right|\left(k_{i} u_{m, n+1}^{2}\right.\right. \\
& \left.-\sum_{s=n+1-k_{i}}^{n} u_{m, s}^{2}\right)+R \sum_{j=1}^{\infty}\left|q_{j}\right|\left[\left(\Delta_{1} u_{m, n+1}\right)^{2}-\left(\Delta_{1} u_{m, n+1-r_{j}}\right)^{2}\right] \\
& \left.+R \sum_{i=1}^{\infty}\left|p_{i}\right|\left[k_{i}\left(\Delta_{1} u_{m, n+1}\right)^{2}-\sum_{s=n+1-k_{i}}^{n}\left(\Delta_{1} u_{m, s}\right)^{2}\right]\right\} \\
= & \sum_{m=0}^{M+1}\left[P Q^{*} u_{m, n+1}^{2}+P P^{\prime} u_{m, n+1}^{2}+R Q^{*}\left(\Delta_{1} u_{m, s}\right)^{2}+R P^{\prime}\left(\Delta_{1} u_{m, n+1}\right)^{2}\right. \\
& -P \sum_{j=1}^{\infty}\left|q_{j}\right| u_{m, n+1-r_{j}}^{2}-P \sum_{i=1}^{\infty}\left|p_{i}\right| \sum_{s=n+1-k_{i}}^{n} u_{m, s}^{2}-R \sum_{j=1}^{\infty}\left|q_{j}\right|\left(\Delta_{1} u_{m, n+1}\right)^{2} \\
& \left.\quad-R \sum_{i=1}^{\infty}\left|p_{i}\right| \sum_{s=n+1-k_{i}}^{n}\left(\Delta_{1} u_{m, s}\right)^{2}\right] .
\end{aligned}
$$

Finally, we take the following Liapunov sequence

$$
V_{n}=V_{n}^{(1)}+V_{n}^{(2)} .
$$

By using (10), we finally get

$$
\Delta V_{n} \leq-2 P\left(1-Q^{*}-\frac{1}{2} P-P^{\prime}\right) \sum_{m=0}^{M+1} u_{m, n+1}^{2} .
$$


Therefore, $\left\{V_{n}\right\}$ is decreasing and has a nonnegative limit of $\left\{V_{n}\right\}$ because $V_{n} \geq 0$ for $n=0,1, \ldots$ Now, summing the two sides of (11) from $n=0$ to $n=\infty$, we have

$$
2 P\left(1-Q^{*}-\frac{1}{2} P-P^{\prime}\right) \sum_{n=0}^{\infty} \sum_{m=0}^{M+1} u_{m, n+1}^{2} \leq V_{0}
$$

or

$$
\sum_{n=0}^{\infty} \sum_{m=0}^{M+1} u_{m, n}^{2} \leq \sum_{m=0}^{M+1} \mu_{m, 0}^{2}+\frac{V_{0}}{2 P\left(1-Q^{*}-\frac{1}{2} P-P^{\prime}\right)}<\infty .
$$

The proof is complete.

Remark 2.1. Let $u_{m, n}$ be independent of $m$ and $x_{n}=u_{m, n}, q_{j}=k_{i}=0$ for $i, j=$ $1,2, \ldots$ and $R=0$. Then (1) becomes an ordinary difference equation:

$$
\Delta x_{n}+P x_{n}=0 \quad \text { for } \quad n=0,1, \ldots,
$$

and (10) becomes

$$
\frac{1}{2} P<1
$$

One can easily prove that the condition (13) is a necessary and sufficient condition for SSS in (12) (in fact, the absolute summable stability, i.e., its solutions $\left\{x_{n}\right\}$ with IC $x_{i}=\mu_{i}$ for $i=\ldots,-1,0$ satisfying

$$
\|\mu\|=\sup \left\{\left|\mu_{i}\right| \text { for } i=\ldots,-1,0\right\}<\infty
$$

has the property: $\left.\sum_{n=0}^{\infty}\left|x_{n}\right|<\infty\right)$. Therefore, in this sense, the condition (10) is a "sharp" condition.

As a special case, we consider a linear parabolic Volterra difference equation of retarded type

$$
\begin{aligned}
& \Delta_{2} u_{m, n}+\sum_{i=1}^{\infty} p_{i} u_{m, n-k_{i}}=R \Delta_{1}^{2} u_{m-1, n+1} \\
& \text { for } m=0,1, \ldots, M-1 \text { and } n=0,1, \ldots,
\end{aligned}
$$

with $\mathrm{NBC}(2)$ and $\mathrm{IC}(3)$. By Theorem 2.1, we have

Corollary 2.1. If

$$
\frac{1}{2} P+P^{\prime}<1
$$

then the zero solution of (14) is SSS.

\section{Equation (4)}

For (4), we have the following

Theorem 3.1. Let (10) be true. And suppose that

$$
f(x) h(x) \geq \max \left\{f^{2}(x), g^{2}(x)\right\}, \quad x \in \mathbf{R},
$$

and

$$
\begin{aligned}
& {[h(y)-h(x)][F(y)-F(x)] \geq} \\
& \quad \max \left\{[F(y)-F(x)]^{2},[g(y)-g(x)]^{2},[f(y)-f(x)]^{2}\right\}
\end{aligned}
$$

for $y, x \in \mathbf{R}$. Then, the zero solution of (4) is $f$-SSS and $g$-SSS. 
Proof. It is easy to show that

$$
\begin{array}{r}
\Delta_{2}\left[h\left(u_{m, n}\right)-\sum_{j=1}^{\infty} q_{j} g\left(u_{m, n-r_{j}}\right)-\sum_{i=1}^{\infty} p_{i} \sum_{s=n-k_{i}}^{n} f\left(u_{m, s}\right)\right] \\
=-P f\left(u_{m, n+1}\right)+R \Delta_{1}^{2} F\left(u_{m-1, n+1}\right) .
\end{array}
$$

One can define a Liapunov sequence as follows

$$
V_{n}^{(1)}=\sum_{m=0}^{M+1}\left[h\left(u_{m, n}\right)-\sum_{j=1}^{\infty} q_{j} g\left(u_{m, n-r_{j}}\right)-\sum_{i=1}^{\infty} p_{i} \sum_{s=n-k_{i}}^{n} f\left(u_{m, s}\right)\right]^{2} .
$$

As in the proof of Theorem 2.1, one gets

$$
\begin{aligned}
\Delta V_{n}^{(1)} \leq & -2 P \sum_{m=0}^{M+1} f\left(u_{m, n+1}\right) h\left(u_{m, n+1}\right)+P\left(Q^{*}+P+P^{\prime}\right) \sum_{m=0}^{M+1} f^{2}\left(u_{m, n+1}\right) \\
& -2 R \sum_{m=0}^{M+1} \Delta_{1} h\left(u_{m, n+1}\right) \Delta_{1} F\left(u_{m, n+1}\right)+R\left(Q^{*}+P^{\prime}\right) \sum_{m=0}^{M+1}\left[\Delta_{1} F\left(u_{m, n+1}\right)\right]^{2} \\
& +P \sum_{m=0}^{M+1} \sum_{j=1}^{\infty}\left|q_{j}\right| g^{2}\left(u_{m, n+1-r_{j}}\right)+P \sum_{m=0}^{M+1} \sum_{i=1}^{\infty}\left|p_{i}\right| \sum_{s=n+1-k_{i}}^{n} f^{2}\left(u_{m, s}\right) \\
& +R \sum_{m=0}^{M+1} \sum_{j=1}^{\infty}\left|q_{j}\right|\left[\Delta_{1} g\left(u_{m, n+1-r_{j}}\right)\right]^{2}+R \sum_{m=0}^{M+1} \sum_{i=1}^{\infty}\left|p_{i}\right| \sum_{s=n+1-k_{i}}^{n}\left[\Delta_{1} f\left(u_{m, s}\right)\right]^{2} .
\end{aligned}
$$

Then, one can take another Liapunov sequence as follows

$$
\begin{aligned}
V_{n}^{(2)}= & \sum_{m=0}^{M+1}\left\{P \sum_{j=1}^{\infty}\left|q_{j}\right| \sum_{s=n+1-r_{j}}^{n} g^{2}\left(u_{m, s}\right)+P \sum_{i=1}^{\infty}\left|p_{i}\right| \sum_{s=n+1-k_{i}}^{n} \sum_{t=s}^{n} f^{2}\left(u_{m, t}\right)\right. \\
& +R \sum_{j=1}^{\infty}\left|q_{j}\right| \sum_{s=n+1-r_{j}}^{n}\left[\Delta_{1} g\left(u_{m, s}\right)\right]^{2} \\
& \left.+R \sum_{i=1}^{\infty}\left|p_{i}\right| \sum_{s=n+1-k_{i}}^{n} \sum_{t=s}^{n}\left[\Delta_{1} f\left(u_{m, t}\right)\right]^{2}\right\} .
\end{aligned}
$$

It follows that

$$
\begin{aligned}
\Delta V_{n}^{(2)}= & \sum_{m=0}^{M+1}\left\{P Q^{*} g^{2}\left(u_{m, n+1}\right)+P P^{\prime} f^{2}\left(u_{m, n+1}\right)+R Q^{*}\left[\Delta_{1} g\left(u_{m, n+1}\right)\right]^{2}\right. \\
& +R P^{\prime}\left[\Delta_{1} f\left(u_{m, n+1}\right)\right]^{2}-P \sum_{j=1}^{\infty}\left|q_{j}\right| g^{2}\left(u_{m, n+1-r_{j}}\right) \\
& -P \sum_{i=1}^{\infty}\left|p_{i}\right| \sum_{s=n+1-k_{i}}^{n} f^{2}\left(u_{m, s}\right)-R \sum_{j=1}^{\infty}\left|q_{j}\right|\left[\Delta_{1} g\left(u_{m, n+1-r_{j}}\right)\right]^{2} \\
& \left.-R \sum_{i=1}^{\infty}\left|p_{i}\right| \sum_{s=n+1-k_{i}}^{n}\left[\Delta_{1} f\left(u_{m, s}\right)\right]^{2}\right\} .
\end{aligned}
$$

Finally, one takes the Liapunov sequence as follows:

$$
V_{n}=V_{n}^{(1)}+V_{n}^{(2)} \text {. }
$$


Then, one obtains from the above and (17)

$$
\begin{aligned}
\Delta V_{n} \leq & \sum_{m=0}^{M+1}\left\{-2 P f\left(u_{m, n+1}\right) h\left(u_{m, n+1}\right)+P\left(Q^{*}+P+P^{\prime}\right) f^{2}\left(u_{m, n+1}\right)\right. \\
& +P Q^{*} g^{2}\left(u_{m, n+1}\right)+P P^{\prime} f^{2}\left(u_{m, n+1}\right)+R Q^{*}\left[\Delta_{1} g\left(u_{m, n+1}\right)\right]^{2} \\
& -2 R \Delta_{1} h\left(u_{m, n+1}\right) \Delta_{1} F\left(u_{m, n+1}\right)+R\left(Q^{*}+P^{\prime}\right)\left[\Delta_{1} F\left(u_{m, n+1}\right)\right]^{2} \\
& \left.+R P^{\prime}\left[\Delta_{1} f\left(u_{m, n+1}\right)\right]^{2}\right\} \\
\leq & \sum_{m=0}^{M+1}\left[-2 P f\left(u_{m, n+1}\right) h\left(u_{m, n+1}\right)\right. \\
& \left.+P\left(Q^{*}+P+2 P^{\prime}\right) f^{2}\left(u_{m, n+1}\right)+P Q^{*} g^{2}\left(u_{m, n+1}\right)\right] \\
= & -2 P \sum_{m=0}^{M+1}\left[\alpha f\left(u_{m, n+1}\right) h\left(u_{m, n+1}\right)-\left(\frac{Q^{*}}{2}+\frac{P}{2}+P^{\prime}\right) f^{2}\left(u_{m, n+1}\right)\right. \\
& \left.+(1-\alpha) f\left(u_{m, n+1}\right) h\left(u_{m, n+1}\right)-\frac{Q^{*}}{2} g^{2}\left(u_{m, n+1}\right)\right] \\
\leq & -2 P \sum_{m=0}^{M+1}\left(\alpha-\frac{Q^{*}}{2}-\frac{P}{2}-P^{\prime}\right) f^{2}\left(u_{m, n+1}\right) \\
& -2 P\left(1-\alpha-\frac{Q^{*}}{2}\right) g^{2}\left(u_{m, n+1}\right) .
\end{aligned}
$$

Then,

$$
\Delta V_{n} \leq-2 P\left(1-Q^{*}-\frac{P}{2}-P^{\prime}\right) \sum_{m=0}^{M+1} f^{2}\left(u_{m, n+1}\right) \quad \text { for } \quad \alpha=1-\frac{Q^{*}}{2}
$$

and

$$
\Delta V_{n} \leq-2 P\left(1-Q^{*}-\frac{P}{2}-P^{\prime}\right) \sum_{m=0}^{M+1} g^{2}\left(u_{m, n+1}\right) \quad \text { for } \quad \alpha=\frac{Q^{*}}{2}+\frac{P}{2}+P^{\prime}
$$

Since $f(x)$ and $g(x)$ are continuous functions and $\|\mu\|<\infty$, one has

$$
\sum_{n=0}^{\infty} \sum_{m=0}^{M+1} \phi^{2}\left(u_{m, n}\right) \leq \sum_{m=0}^{M+1} \phi^{2}\left(\mu_{m, 0}\right)+\frac{V_{0}}{2 P\left(1-Q^{*}-\frac{1}{2} P-P^{\prime}\right)}<\infty
$$

where $\phi(x)=f(x)$ or $\phi(x)=g(x)$. This completes the proof.

We now give two corollaries.

Consider the nonlinear parabolic Volterra difference equation of neutral type:

$$
\begin{gathered}
\Delta_{2}\left[u_{m, n}-\sum_{j=1}^{\infty} q_{j} g\left(u_{m, n-r_{j}}\right)\right]+\sum_{i=1}^{\infty} p_{i} f\left(u_{m, n-k_{i}}\right)=R \Delta_{1}^{2} u_{m-1, n+1} \\
\text { for } m=0,1, \ldots, M-1 \text { and } n=0,1, \ldots,
\end{gathered}
$$

with $\mathrm{NBC}(2)$ and $\mathrm{IC}(3)$. By using Theorem 3.1, we obtain the following 
Corollary 3.1. Let (10) be true, and assume that

$$
x f(x) \geq \max \left\{f^{2}(x), g^{2}(x)\right\}, \quad x \in \mathbf{R},
$$

and that

$$
(y-x)^{2} \geq \max \left\{[f(y)-f(x)]^{2},[g(y)-g(x)]^{2}\right\}, \quad y, x \in \mathbf{R} .
$$

Then, the zero solution of (18) is $f$-SSS and $g$-SSS.

More specifically, we consider the nonlinear parabolic Volterra difference equation of retarded type:

$$
\begin{aligned}
& \Delta_{2} u_{m, n}+\sum_{i=1}^{\infty} p_{i} f\left(u_{m, n-k_{i}}\right)=R \Delta_{1}^{2} u_{m-1, n+1} \\
& \text { for } m=0,1, \ldots, M-1 \text { and } n=0,1, \ldots,
\end{aligned}
$$

with $\mathrm{NBC}(2)$ and IC(3). Again, by Theorem 3.1, we get

Corollary 3.2. Let (15) be true, and assume that

$$
x f(x) \geq f^{2}(x), \quad x \in \mathbf{R},
$$

and that

$$
(y-x)^{2} \geq[f(y)-f(x)]^{2}, \quad y, x \in \mathbf{R} .
$$

Then, the zero solution of (21) is $f$-SSS.

\section{An example}

Consider the generalized first equation of Open Problem 6.8.1 in Kocic and Ladas [12]:

$$
\begin{gathered}
\Delta_{2} u_{m, n}+\sum_{i=1}^{\infty} p_{i}\left[\exp \left(u_{m, n-k_{i}}\right)-1\right]=R \Delta_{1}^{2} u_{m-1, n+1} \\
\text { for } m=0,1, \ldots, M-1 \text { and } n=0,1, \ldots
\end{gathered}
$$

and

$$
\begin{gathered}
\Delta_{2} u_{m, n}+\sum_{i=1}^{\infty} p_{i}\left[1-\exp \left(-u_{m, n-k_{i}}\right)\right]=R \Delta_{1}^{2} v_{m-1, n+1} \\
\quad \text { for } m=0,1, \ldots, M-1 \text { and } n=0,1, \ldots,
\end{gathered}
$$

with $\mathrm{NBC}(2)$ and $\mathrm{IC}(3)$, respectively.

For (24) (resp. (25)), we know that $f(x)=e^{x}-1$ (resp. $f(x)=1-e^{-x}$ ). It is easy to prove that (22) (resp. (23)) is satisfied for $x<0$ (resp. $x>0$ ). Hence, we can choose $\phi(x)=e^{x}-1$ (resp. $\phi(x)=1-e^{-x}$ ) which satisfies

$$
\phi(x)=0 \text { implies } x=0 .
$$

Hence, if (15) is true, then every negative (or positive) solution which satisfies (6) must satisfy (7) where a negative (or positive) solution means $\mu_{m, i}, u_{m, n}<$ (or $>$ ) 0 for $m=0,1, \ldots, M-1, i=\ldots,-1,0$, and $n=0,1, \ldots$

Combining (24) with (25), we consider the following equation:

$$
\begin{gathered}
\Delta_{2} u_{m, n}+\sum_{i=1}^{\infty} p_{i}\left[1-\exp \left(-u_{m, n-k_{i}} \operatorname{sgn} u_{m, n-k_{i}}\right)\right] \operatorname{sgn} u_{m, n-k_{i}}=R \Delta_{1}^{2} u_{m-1, n+1} \\
\text { for } m=0,1, \ldots, M-1 \text { and } n=0,1, \ldots,
\end{gathered}
$$

with $\mathrm{NBC}(2)$ and $\mathrm{IC}(3)$ and obtain the following result. 
Theorem 4.1. If (15) holds, then the zero solution of (26) is AS.

\section{Appendix}

On the (unique) existence of solutions of initial-boundary value problem (1), (2), and (3): rewrite (1) as

$$
\begin{aligned}
u_{m, n+1}-u_{m, n} & -\sum_{j=1}^{\infty} q_{j} u_{m, n+1-r_{j}}+\sum_{j=1}^{\infty} q_{j} u_{m, n-r_{j}}+\sum_{i=1}^{\infty} p_{i} u_{m, n-k_{i}} \\
& =R \Delta_{1} u_{m, n+1}-R \Delta_{1} u_{m-1, n+1} \\
& =R\left(u_{m+1, n+1}-2 u_{m, n+1}+u_{m-1, n+1}\right) .
\end{aligned}
$$

We may assume that $R \neq 0$ and $r_{j} \in\{1,2, \ldots\}$. Then, for $m=0$ and $n=0$, we have $\Delta_{1} u_{0,1}=0$, so

$$
u_{0,1}-u_{0,0}-\sum_{j=1}^{\infty} q_{j} u_{0,1-r_{j}}+\sum_{j=1}^{\infty} q_{j} u_{0,-r_{j}}+\sum_{i=0}^{\infty} p_{i} u_{0,-k_{i}}=-R u_{0,1} .
$$

It follows that

$$
u_{0,1}=\frac{1}{1+R}\left(u_{0,0}+\sum_{j=1}^{\infty} q_{j} u_{0,1-r_{j}}-\sum_{j=1}^{\infty} q_{j} u_{0,-r_{j}}-\sum_{i=0}^{\infty} p_{i} u_{0,-k_{i}}\right)
$$

and

$$
u_{1,1}=u_{0,1} \text {. }
$$

For $m=1$ and $n=0$, we have $\Delta_{1} u_{0,1}=0$, so

$$
u_{2,1}=\frac{1}{R}\left(u_{1,1}-u_{1,0}-\sum_{j=1}^{\infty} q_{j} u_{1,1-r_{j}}+\sum_{j=1}^{\infty} q_{j} u_{1,-r_{j}}+\sum_{i=1}^{\infty} p_{i} u_{1,-k_{i}}\right)+u_{1,1} .
$$

For $m=M-1$ and $n=0$, we have

$$
\begin{aligned}
u_{M, 1}= & \frac{1}{R}\left(u_{M-1,1}-u_{M-1,0}-\sum_{j=1}^{\infty} q_{j} u_{M-1,1-r_{j}}+\sum_{j=1}^{\infty} q_{j} u_{M-1,-r_{j}}\right. \\
& \left.+\sum_{i=1}^{\infty} p_{i} u_{M-1,-k_{i}}\right)+2 u_{M-1,1}-u_{M-2,1} .
\end{aligned}
$$

Finally, for $m=M$ and $n=0$, we have $\Delta_{1} u_{M, 1}=0$, so we have

$$
u_{M+1,1}=u_{M, 1} \text {. }
$$

In this way, we can successively calculate

$$
u_{0,2}, \ldots, u_{M, 2}, u_{M+1,2}, u_{0,3}, \ldots, u_{M, 3}, u_{M+1,3}, \ldots
$$

Acknowledgments. The authors would like to thank referees for their valuable suggestions and comments. This project is supported by the National Natural Science Foundation of China. 


\section{References}

1. D. D. Bainov, A. D. Myshkis, and A. I. Zahariev, On the oscillating properties of the solutions of a class of integrodifferential equations of neutral type, Int. J. Math. \& Math. Sci. 15 (1992), $119-128$.

2. T. A. Burton, Volterra Integral and Differential Equations, Academic Press, New York, 1983.

3. L_ Stability and Periodic Solutions of Ordinary and Functional Differential Equations, Academic Press, New York, 1985.

4. B. T. Cui, Oscillation theorems of nonlinear parabolic equations of neutral type, Math. J. Toyama Univ. 14 (1991), 113-123.

5. _ Oscillation properties for parabolic equations of neutral type, Comment. Math. Univ. Carolinae 33 (1992), 581-588.

6. __ Oscillation theorems for nonlinear hyperbolic equations with deviating arguments, Acta Sci. Math. (Szeged) 58 (1993), 159-168.

7. S. Elaydi, Periodicity and stability of linear Volterra difference systems, J. Math. Anal. Appl. 181 (1994), 483-492.

8. K. Gopalsamy and P. X. Weng, On the stability of a neutral integro-partial differential equation, Bull. Inst. Math. Acad. Sinica 20 (1992), 267-284.

9. T. Hara, T. Yoneyama, and T. Itoh, Asymptotic stability criteria for nonlinear Volterra integrodifferential equations, Funkcial. Ekvac. 33 (1990), 39-57.

10. Y. Hino and S. Murakami, Stability properties of Volterra equations, J. Diff. Eqns. 89 (1991), 121-137.

11. W. G. Kelley and A. C. Peterson, Difference Equations: An Introduction with Applications, Academic Press, New York, 1991.

12. V. L. Kocic and G. Ladas, Global Behavior of Nonlinear Equations of Higher Order with Applications, Kluwer Acad. Publ., Dordrecht, 1993.

13. A. H. Nasr, Asymptotic behavior and oscillation of classes of integro-differential equations, Proc. Amer. Math. Soc. 116 (1992), 143-148.

14. B. Shi, Z. C. Wang, and J. S. Yu, Square summable stability and existence of positive solutions in neutral Volterra difference equations, to appear.

15. M. Q. Wang, L. Wang, and X. T. Du, Stability of Volterra integral and differential equations, Acta Math. Appl. Sinica 15 (1992), 184-193.

16. S. L. Xie, Stability of solutions of a class of parabolic partial funciional differential equations with deviating arguments, Chin. Sci. Bull. 36 (1991), 1435-1436.

17. N. Yoshida, On the zeros of solutions of hyperbolic equations of neutral type, Diff. Int. Eqns. 3 (1990), 155-160.

18. J. S. Yu and Z. C. Wang, Asymptotic behavior and oscillation in delay difference equations, Funkcial. Ekvac. 37 (1994), 241-248.

19. J. S. Yu and S. S. Cheng, A stability criterion for a neutral difference equation with delay, Appl. Math. Lett. 7 (1994), 75-80.

20. Y. H. Yu and B. T. Cui, Advances in the oscillation theory of functional partial differential equations, in Theory and Applications of Ordinary Differential Equations (Eds. L. Wang, et al.), Sci. Press, 1992, pp.38-41.

21. B. G. Zhang, Forced oscillation of neutral equations, Chin. Sci. Bull. 34 (1989), 1845-1848.

22. B. G. Zhang, S. T. Liu, and S. S. Cheng, Oscillation of a class of delay partial difference equations, J. Diff. Eqns. and Appl. 1 (1995), 215-226.

Department of Applied Mathematics, Hunan University, Changsha, Hunan, 410082, P. R. CHINA 